\title{
Impacto na saúde humana de disruptores endócrinos presentes em corpos hídricos: existe associação com a obesidade?
}

\author{
Impact on human health of endocrine disruptors present in \\ environmental water bodies: is there an association with obesity?
}

Regina Célia Nucci Pontelli ${ }^{1}$

Altacilio Aparecido Nunes ${ }^{1}$

Sonia Valle Walter Borges de Oliveira ${ }^{2}$

${ }^{1}$ Departamento de Medicina Social, Faculdade de Medicina de Ribeirão Preto, Universidade de São Paulo. Av. Bandeirantes 3900, Campus USP. 14049900 Ribeirão Preto SP Brasil. altacilio@fmrp.usp.br ${ }^{2}$ Departamento

de Administração, Faculdade de Economia, Administração e Contabilidade de Ribeirão Preto, Universidade de São Paulo. Ribeirão Preto SP Brasil.

\begin{abstract}
There is growing evidence that endocrine disruptors (ED) may adversely affect humans. Surface and underground water are the main sources for obtaining potable water, however they can be contaminated with ED, which are not completely removed by conventional water and sewage treatment processes. Some health problems are related to the exposure of humans to $E D$, obesity being one of them. There is currently an increase in the prevalence of obesity worldwide, a fact that is considered a concern in view of its potential impact on the health care system, since obesity is the major risk factor of the leading chronic diseases including diabetes and cardiovascular disease. By means of a review of the literature, this paper sought to gather scientific publications linking exposure to ED with obesi$t y$, in order to verify the importance of removal of ED from water bodies, thereby preserving the population's health and aquatic biota. Most of the selected studies suggest an association between ED and obesity in humans.
\end{abstract}

Key words Endocrine disruptors, Environment, Water, Obesity
Resumo Há evidências crescentes de que os desreguladores endócrinos (DE) podem afetar negativamente os seres humanos. Águas superficiais e subterrâneas são as principais fontes de obter a sua potabilidade, no entanto, podem ser contaminadas por DE, que não são totalmente removidos pelos processos convencionais de tratamento de água e esgoto. Alguns problemas de saúde estão relacionados à exposição dos seres humanos aos DE, sendo a obesidade um deles. Atualmente vive-se um aumento da prevalência da obesidade em todo o mundo, fato considerado preocupante, tendo em vista seu potencial impacto no sistema de saúde, pois, as principais doenças crônicas como diabetes e doenças cardiovasculares, têm a obesidade como grande fator de risco. Por meio de revisão de literatura, este artigo buscou reunir publicações científicas relacionando a exposição aos DE com a obesidade, com o objetivo de verificar a importância de sua remoção dos corpos hídricos, preservando a saúde da população e da biota aquática. A maioria dos estudos selecionados sugere associação entre DE e obesidade em humanos.

Palavras-chave Disruptores endócrinos, Meio ambiente, Água, Obesidade 


\section{Introdução}

Os corpos hídricos superficiais e subterrâneos são os principais reservatórios de água potável, podendo facilmente ser contaminados pela infiltração de substâncias químicas através do solo, dos produtos utilizados na agricultura e pecuária, de excretas de animais, ou através dos efluentes industriais e domésticos, pois, de maneira geral, essas substâncias não são removidas pelos processos convencionais de tratamento de água e esgoto ${ }^{1}$.

O Brasil ocupa uma posição confortável em relação à disponibilidade hídrica, com $12 \%$ da quantidade mundial, porém, a água disponível se encontra distribuída de maneira desigual, ocorrendo maior abundância em locais com menores índices demográficos, ao contrário do que seria uma situação ideal ${ }^{2}$. Nas regiões com menor disponibilidade dos recursos hídricos ocorre maior probabilidade de contaminação devido à densidade demográfica mais elevada e à presença de grandes polos industriais.

Grande parte dos efluentes urbanos no Brasil chega aos corpos hídricos sem tratamento, portanto, diariamente é despejada no meio ambiente uma carga excessiva de esgotos in natura, sejam eles provenientes da captação ou descartados de maneira irregular, e que contaminam o solo e os corpos hídricos com impactos diretos na saúde da população. A média de coleta de esgoto dos municípios brasileiros é de $62,54 \%$, sendo que destes, apenas $39 \%$ possuem tratamento antes de serem despejados nos corpos hídricos, dados bem abaixo dos 95\% necessários para se obter a universalização de tratamento ${ }^{3}$. Os efluentes de Estações de Tratamento de Esgotos (ETE) contêm um grande número de contaminantes, alguns identificados, outros desconhecidos; porém, em ambos os casos nem todos estão contemplados na legislação brasileira, embora necessitem ser detectados, avaliados com relação a suas características físico-químicas, monitorados e, idealmente, removidos devido a sua capacidade de causarem efeitos adversos à biota aquática e ao homem ${ }^{4}$. Fazem parte desse grupo de contaminantes um conjunto específico de substâncias químicas capazes de simular ou alterar o sistema hormonal de seres humanos e animais, prejudicando o funcionamento normal dos sistemas imunológico, nervoso e endócrino, denominados interferentes endócrinos, disruptores endócrinos ou desreguladores endócrinos, entre outras terminologias.

\section{Disruptores endócrinos}

O termo foi usado pela primeira vez na Conferência Wingspread, EUA, em 1991, para nomear substâncias que ao interferirem no sistema endócrino, causam efeitos adversos à saúde e à biota $^{6}$. A Agência Europeia de Ambiente (EEA), em 2012, definiu DE como "substâncias exógenas que causam efeitos adversos na saúde de um organismo intacto e sua descendência, resultantes de alterações na função endócrina"7. As substâncias classificadas como DE possuem diferentes configurações químicas e são produzidas naturalmente pelo organismo, como o caso dos estrogênios ou são produzidas sinteticamente ${ }^{8}$. Os mecanismos pelos quais essas substâncias agem nos organismos vivos ainda não foram totalmente elucidados, mas fatores como dose, duração de contato e via de exposição podem interferir na sua ação $0^{9-12}$.

\section{Efeitos da exposição aos DE}

A preocupação com os DE foi originalmente associada à ocorrência de distúrbios em várias espécies selvagens. Dentre as observações iniciais foram relatados casos de fertilidade reduzida, anomalias no sistema reprodutivo e distúrbios comportamentais. Em mulheres que fizeram uso do Estrógeno Sintético Dietiestilbestrol (DES), utilizado para prevenir abortos espontâneos entre as décadas de 40 a 70, foram observados casos de câncer nos órgãos reprodutivos de seus descendentes. O DES serviu como um modelo para pesquisas no qual a exposição a um DE durante a fase gestacional pode afetar os descendentes na vida adulta, devido à estimulação da atividade mitótica principalmente dos tecidos do trato genital feminino, apesar de afetar outros tecidos, inclusive dos machos ${ }^{13,14}$.

Atualmente o aumento da prevalência de distúrbios como obesidade, diabetes, hipotireoidismo, déficit de atenção, autismo e distúrbios respiratórios, está sendo evidenciado em várias pesquisas que relacionam tais problemas com a exposição aos $\mathrm{DE}^{15-19}$.

\section{DE e corpos hídricos}

Os tratamentos convencionais de água e esgoto sanitário não são capazes de remover completamente tais substâncias, proporcionando a permanência das mesmas no meio aquático ${ }^{20-23}$. Mesmo estando presentes em baixas concentrações, na ordem de nanograma por litro, são capa- 
zes de apresentar atividade biológica com efeitos deletérios no homem ${ }^{20}$. Existe a necessidade de processos mais eficazes que façam a remoção ou a desativação da atividade biológica dessas substâncias.

As ETE foram projetadas para reduzir a carga de poluentes orgânicos, nutrientes e microrganismos patogênicos, não objetivando especificamente a remoção dos DE presentes no esgoto $^{24}$. Atualmente, os processos oxidativos vêm ganhando atenção no tratamento de efluentes industriais e domésticos, através de tecnologias promissoras de remoção, que incluem processos oxidativos avançados (POA), ozonização associada a peróxido de hidrogênio $\left(\mathrm{O}_{3} / \mathrm{H}_{2} \mathrm{O}_{2}\right)$ e fotocatálise $\left(\mathrm{H}_{2} \mathrm{O}_{2} / \mathrm{UV}\right)^{25}$. Outros tratamentos também estão sendo pesquisados em sistemas aquosos, como adsorção em carvão ativado, nanofiltração em membranas, osmose reversa, cloração e reatores com lâmpadas $\mathrm{UV}^{26}$. Do ponto de vista econômico, a correta destinação e o tratamento do esgoto reduzem os gastos relacionados aos processos de tornar a água potável e as despesas com saúde pública e, em termos de meio ambiente, contribui para a diminuição da poluição dos corpos hídricos ${ }^{27}$.

\section{DE e obesidade}

Dentre os efeitos deletérios que os DE podem causar, a relação entre a exposição e a obesidade se torna preocupante, uma vez que tem potencial para sobrecarregar sistemas de saúde em todo o mundo. Os distúrbios associados à exposição como diabetes e doenças cardiovasculares se apresentam em proporções epidêmicas, tornando-se uma ameaça crescente à saúde pública mundial ${ }^{28}$. Atualmente, existem estudos suficientes que comprovam o potencial obesogênico dos DE, em especial no que se refere à exposição durante o período intrauterino ${ }^{29}$. Obesogênicos são produtos químicos, ou xenobióticos naturais, que promovem a obesidade, aumentando o número de células adiposas, alterando o armazenamento de lipídios em células adiposas preexistentes, interferindo nos mecanismos através do qual o corpo regula o apetite e a saciedade ${ }^{30,31}$. Um dos primeiros pesquisadores a questionar a hipótese de existir uma relação entre a obesidade e o DE foi Baillie-Hamilton ${ }^{32}$, ao publicar um estudo analisando essa correlação.

Atualmente, pesquisas realizadas com testes in vitro ou com animais de laboratório e estudos epidemiológicos vinculam a exposição humana aos DE à obesidade, à síndrome metabólica e ao diabetes tipo $2^{33}$. A maioria dessas pesquisas incluem os DE: DES, bisfenol A (BPA), Diclorodifeniltricloroetano (DDT) e seus metabolitos, Tributil-estanho (TBT) e ftalatos, todos relacionados à atividade obesogênica ${ }^{34}$. $\mathrm{O}$ mecanismo de ação dos DE relacionados à obesidade necessitam ser melhor elucidados, porém pesquisas apontam que essas substâncias podem se ligar diretamente aos receptores hormonais nucleares que agem na regulação da diferenciação e proliferação de adipócitos, ou impactar o metabolismo e transporte de hormônios endógenos ${ }^{35}$. O PPAR (receptor ativado por proliferadores de peroxissoma gama) é um tipo de receptor nuclear que atua no metabolismo de adipócitos sugerindo uma ação direta entre os agonistas do PPAR e a indução da adipogênese ${ }^{36}$. Outro receptor importante na regulação da adipogênese, bem como para a homeostase energética, é o receptor de glicocorticoide $(\mathrm{RG})^{37}$. O mecanismo de ação envolve ainda outros fatores. Verificou-se que agem em doses diminutas com efeito não linear em relação à dose, e que podem estar relacionados com a programação epigenética. Alterações epigenéticas são sensíveis a modificações ambientais que podem causar mudanças transmitidas aos descendentes, como a obesidade ${ }^{38-40}$. Segundo Tang e $\mathrm{Ho}^{41}$ a epigenética é definida como mudanças na expressão do gene que não alteram a sequência do DNA, mas que são herdáveis ao longo das gerações. Existem fatos convincentes para sugerir que a presença de produtos químicos no meio ambiente é um fator que contribui para a epidemia de obesidade, embora ainda seja desconhecido o mecanismo pelo qual eles influenciam a obesidade em seres humanos ${ }^{30}$.

\section{Metodologia}

Para o presente estudo foi definido como problema a relação entre a presença de DE no ambiente, com potencial efeito na obesidade, e a necessidade de se evitar a contaminação dos corpos hídricos por esses contaminantes. Realizou-se um levantamento bibliográfico sistematizado sobre as evidências científicas existentes relativas ao tema, conduzido por meio de um protocolo de busca de artigos publicados entre janeiro/2010 e maio/2015, nas bases de dados: PubMed, Scopus, Lilacs e Web of Science, utilizando os termos "Endocrine Disruptor", “Environmental”, "Obesity”, nos idiomas Português, Inglês e Espanhol.

Para a seleção dos artigos foram utilizados os critérios: a) artigo contemplando a correlação 
entre DE e obesidade; b) somente estudos primários; c) acesso ao artigo na íntegra. Foram excluídos os artigos de revisão, que foram utilizados somente para contextualização do tema. A inclusão dos artigos obtidos na busca foi realizada por dois pesquisadores independentes.

\section{Resultados}

A busca resultou em 343 artigos, dos quais foram retirados aqueles em duplicata, e posteriormente selecionados pelo título e resumo, resultando em 165. A aplicação do teste de relevância resultou em 46 artigos que foram analisados através da leitura na íntegra. Nessa fase, foram selecionados 37 artigos por apresentarem dados mais conclusivos, demonstrando uma relação positiva entre a exposição e a obesidade. Apesar dos 46 artigos responderem de forma afirmativa ao teste de relevância, foram excluídos aqueles cujos resultados não se mostraram claramente conclusivos, que abordaram DE cuja exposição não ocorre habitualmente ou cujas estimativas de risco da exposição não foram devidamente relatadas.

Nos Quadros 1 e 2, os artigos selecionados podem ser observados. Eles relatam estudos epidemiológicos, de experimentação animal e com testes in vitro, respectivamente. Foram selecionados estudos epidemiológicos (caso controle, coorte e transversais) que apesar de não apresentarem exposições controladas como os testes com experimentação animal e in vitro, são importantes para o entendimento da relação DE e obesidade. Os estudos foram conduzidos com adultos, crianças e filhos de mães expostas durante a gestação para a obtenção de dados sobre a obesidade possivelmente causada pela exposição aos DE.

Como pode ser observada, a maioria dos 18 estudos observacionais realizados e aqui selecionados sugere a associação entre os vários DE e a ocorrência de obesidade.

\section{Bisfenol}

$\mathrm{O}$ representante mais importante da classe dos bisfenóis é o BPA, um xenoestrógeno, de caráter lipofílico, com poder de bioacumulação no tecido adiposo. Como componente principal do plástico, é encontrado em garrafas de água, nas embalagens de alimentos e produtos dentários ${ }^{7}$. Dentre os DE pesquisados, a exposição ao BPA foi a mais analisada. O BPA é um produto químico de elevado volume de produção, que foi detectado em $93 \%$ da população dos Estados Unidos ${ }^{42}$.
Estudos epidemiológicos: a exposição ao BPA, detectada pela sua concentração na urina, foi associada ao excesso de peso em crianças de 4 a 12 anos na China, mostrando uma relação dose -resposta com o aumento do seu nível na urina, associada com aumento do risco de obesidade para meninas entre 9 e 12 anos. Nesse estudo, a relação não foi positiva entre meninos ${ }^{43}$. Nos EUA, no Vale de Salinas, um estudo de coorte acompanhou crianças nascidas entre 2000 e 2002 desde a fase gestacional até os dias atuais. Os estudos inicialmente foram realizados para analisar os efeitos de pesticidas nos descendentes, porém, atualmente ocorre o biomonitoramento de vários DE. O estudo que leva o nome de C.H.A.M.A.C.O.S (Center for the Health Assessment of Mothers and Children of Salinas) iniciou com agricultoras da região, perfazendo o total de 601 mães e 536 crianças nascidas e inseridas no programa. Aos 9 anos de acompanhamento, 336 dessas crianças ainda faziam parte do programa. Em 2011, mais 300 crianças da região com a mesma idade se uniram ao grupo ${ }^{44}$. Dentre os artigos selecionados, Harley et al. ${ }^{45}$ e Volberg et al. ${ }^{42}$ utilizaram dados desse estudo para analisar se as concentrações pré-natal e pós-natal urinárias de BPA poderiam estar associadas com índice de massa corporal (IMC), circunferência da cintura, porcentagem de gordura corporal e obesidade em algumas dessas crianças. Para o primeiro autor, as concentrações mais elevadas de BPA na urina das crianças aos 9 anos de idade foram associadas à maior chance de obesidade e ao aumento do IMC, circunferência da cintura e percentual de gordura corporal. $\mathrm{O}$ segundo autor analisou a relação entre as concentrações urinárias de BPA pré-natal com taxas de hormônios relacionados à obesidade nas crianças aos 9 anos de idade. As concentrações urinárias de BPA foram medidas em duas fases da gestação, no início $(12,6 \pm 3,9$ semanas) e no final $(26,3 \pm 2,5$ semanas $)$, e as conclusões obtidas foram que as concentrações elevadas no final da gestação estiveram associadas com o aumento da leptina plasmática em meninos, enquanto que no início da gravidez foram associadas com os níveis de adiponectina plasmática aumentada em meninas. Diferenças entre os sexos não foram detectadas no resultado de um estudo realizado com 587 crianças cujos pais foram expostos a BPA de maneira ocupacional. O resultado foi a constatação de que existe relação entre exposição gestacional ao BPA e baixo peso dos descendentes ao nascer ${ }^{46}$. Nos EUA, uma análise transversal foi realizada visando obter dados sobre as concentrações de BPA uri- 
Quadro 1. Características gerais dos estudos epidemiológicos selecionados.

\begin{tabular}{|c|c|c|c|}
\hline Autor & País & Estudo & $\begin{array}{l}\text { Relação entre exposição } \\
\text { aos DEs e obesidade }\end{array}$ \\
\hline Carwile \& Michels (2011) ${ }^{47}$ & EUA & Transversal & Aumento da população de obesos nos EUA \\
\hline Choi et al. $(2014)^{48}$ & Coreia do Sul & Transversal & $\begin{array}{l}\text { Desenvolvimento de obesidade infantil em } \\
\text { meninas }\end{array}$ \\
\hline Elobeid et al. $(2010)^{49}$ & EUA & Transversal & Aumento do índice de massa corporal \\
\hline Gauthier et al. $(2014)^{50}$ & Canadá & Transversal & Níveis elevados de DEs no sangue de obesos \\
\hline Harley et al. $(2013)^{45}$ & USA & Transversal & Aumento do IMC em crianças de 9 anos de idade \\
\hline Hatch et al. $(2015)^{51}$ & USA & Transversal & $\begin{array}{l}\text { Associação entre exposição pré-natal e obesidade } \\
\text { em mulheres }\end{array}$ \\
\hline Li et al. $(2013)^{43}$ & China & Transversal & Associação positiva com obesidade infantil \\
\hline Miao et al. $(2011)^{46}$ & EUA & Coorte & $\begin{array}{l}\text { Exposição intrauterina associada ao baixo peso } \\
\text { ao nascer }\end{array}$ \\
\hline Pestana et al. $(2014)^{52}$ & Portugal & Coorte & $\begin{array}{l}\text { Relação positiva entre exposição e síndrome } \\
\text { metabólica }\end{array}$ \\
\hline Rönn et al. $(2014)^{53}$ & Suécia & Coorte & Alterações no controle da fome e da saciedade \\
\hline Rundle et al. $(2012)^{54}$ & EUA & Coorte & $\begin{array}{l}\text { Exposição pré-natal e aumento de tecido adiposo } \\
\text { durante a infância }\end{array}$ \\
\hline Savastano et al. $(2015)^{55}$ & Itália & Transversal & Aumento da obesidade visceral \\
\hline Scinicariello \& Buser $(2014)^{56}$ & EUA & Transversal & $\begin{array}{l}\text { Relação positiva entre exposição e obesidade em } \\
\text { crianças }\end{array}$ \\
\hline Vilahur et al. $(2013)^{57}$ & Espanha & Coorte & $\begin{array}{l}\text { Exposição pré-natal e aumento do peso de } \\
\text { machos ao nascer }\end{array}$ \\
\hline Volberg et al. $(2013)^{42}$ & EUA & Coorte & $\begin{array}{l}\text { Aumento da leptina plasmática em meninos e } \\
\text { adiponectina em meninas }\end{array}$ \\
\hline Warner et al. $(2014)^{58}$ & EUA & Coorte & Aumento da obesidade apenas em meninos \\
\hline Wohlfahrt-Veje et al. $(2011)^{59}$ & Dinamarca & Coorte & $\begin{array}{l}\text { Exposição ocupacional materna associada com } \\
\text { menor peso da criança ao nascer }\end{array}$ \\
\hline Xue et al. $(2015)^{60}$ & EUA & Caso controle & $\begin{array}{l}\text { Dentre os } 11 \text { DEs estudados, apenas um } \\
\text { apresentou relação com obesidade }\end{array}$ \\
\hline
\end{tabular}

nária, o IMC e a circunferência da cintura em 2.747 pessoas com idade entre 18-74 anos, usando dados obtidos do programa National Health and Nutrition Examination Surveys (NHANES), entre 2003 e 2006. O estudo mostra a possível relação entre a exposição ao BPA e a obesidade da população adulta nos EUA ${ }^{47}$. Outro estudo transversal, realizado em 76 adultos masculinos ambientalmente expostos a BPA na Itália, observou que tanto os níveis de citocinas pró-inflama- 
Quadro 2. Características gerais dos estudos experimentais selecionados.

\begin{tabular}{|c|c|c|}
\hline Autor & País & Relação entre exposição aos DEs e obesidade \\
\hline Bastos Sales et al. $(2013)^{61}$ & Holanda & Alterações funcionais na diferenciação de adipócitos \\
\hline Biemann et al. $(2014)^{62}$ & Alemanha & $\begin{array}{l}\text { Desenvolvimento de adipócitos e a expressão de genes } \\
\text { marcadores de adipogênese }\end{array}$ \\
\hline Hao et al. $(2012)^{63}$ & China & Indução na diferenciação de adipócitos e a adipogênese \\
\hline Hao et al. $(2012)^{64}$ & China & Aumento de tecido adiposo, lipídeos e glicose \\
\hline Hao et al. $(2012)^{65}$ & China & Aumento de tecido adiposo, lipídeos e glicose \\
\hline Hao at al. $(2013)^{66}$ & China & Aumento de tecido adiposo, lipídeos e glicose \\
\hline Kamstra et al. $(2014)^{67}$ & Holanda & Indução na diferenciação de adipócitos por diversos mecanismos \\
\hline Lyche et al. $(2011)^{68}$ & Noruega & $\begin{array}{l}\text { Alterações nos genes responsáveis pela homeostase do peso e } \\
\text { sinalização da insulina }\end{array}$ \\
\hline Marmugi et al. $(2014)^{69}$ & França & $\begin{array}{l}\text { Exposições na fase adulta e perinatal com alterações de ganho } \\
\text { de peso }\end{array}$ \\
\hline Neel et al. $(2013)^{70}$ & EUA & $\begin{array}{l}\text { Produção de glicocorticoides alterada com aumento de } \\
\text { distúrbios metabólicos }\end{array}$ \\
\hline Pereira-Fernandes et al. $(2013)^{71}$ & Bélgica & Propriedade obesogênica confirmada \\
\hline Sargis et al. $(2010)^{72}$ & EUA & $\begin{array}{l}\text { Ativação da adipogênese através da estimulação de receptores } \\
\text { glicocorticoides }\end{array}$ \\
\hline Schmidt et al. $(2012)^{73}$ & Alemanha & Diminuição da fertilidade e aumento de gordura visceral \\
\hline Skinner et al. $(2013)^{74}$ & EUA & $\begin{array}{l}\text { Indução da obesidade e alterações na próstata e ovários devido a } \\
\text { herança transgeracional }\end{array}$ \\
\hline Tracey et al. $(2013)^{75}$ & EUA & $\begin{array}{l}\text { Aumento da obesidade e aumento da incidência de distúrbios } \\
\text { renais de próstata e ovários }\end{array}$ \\
\hline Van Esterik et al. $(2011)^{76}$ & Holanda & $\begin{array}{l}\text { Exposição perinatal induziu aumento de peso corporal somente } \\
\text { em machos }\end{array}$ \\
\hline Wei et al. $(2011)^{77}$ & China & Desenvolvimento de síndrome metabólica na idade adulta \\
\hline Werner Fürst et al. $(2012)^{78}$ & Alemanha & $\begin{array}{l}\text { Relacionou exposição a estrogênios exógenos ao aumento de } \\
\text { tecido adiposo }\end{array}$ \\
\hline Xu et al. $(2011)^{79}$ & Japão & Aumento da tendência de ingerir doces \\
\hline
\end{tabular}

tórias, quanto de BPA foram significativamente maiores em indivíduos com adiposidade visceral $^{55}$. Praticamente na mesma faixa etária, 890 pessoas entre homens e mulheres de 70 anos de idade foram analisadas para verificar a associação entre BPA e IMC, distribuição de gordura e níveis circulantes de adiponectina, leptina e grelina. Os resultados levaram à hipótese de que as taxas de 
BPA foram associadas positivamente com os níveis de adiponectina e leptina, mas negativamente com a grelina. De acordo com o estudo, o BPA pode interferir no controle hormonal da fome e da saciedade ${ }^{54}$. Na Índia, um estudo com crianças pesquisou a presença na urina de 26 tipos diferentes de DE, sendo encontradas 11 substâncias, incluindo BPA, porém não foram conclusivas as associações com a obesidade, apenas foi observado que as concentrações encontradas estavam acima dos níveis observados em estudos similares nos EUA e China ${ }^{60}$.

Estudos experimentais: algumas pesquisas foram realizadas expondo camundongos na idade gestacional e os resultados confirmaram a correlação entre exposição ao BPA com aumento do peso corporal em machos através de alterações nos tecidos analisados ${ }^{76}$. Quando animais de laboratório são expostos ao BPA e simultaneamente são apresentados a uma dieta rica em gorduras logo após o desmame, a alta taxa de gordura pode ser um gatilho para acelerar os efeitos adversos ocasionados pela exposição ao $\mathrm{BPA}^{77}$. Por considerar que estudos experimentais focam preferencialmente exposições de curta duração, Marmugi et al. ${ }^{69}$ investigaram o efeito de oito meses de exposição de BPA nos marcadores metabólicos hepáticos e no plasma em ratos adultos. Os testes revelaram uma hipercolesterolemia devida a um aumento na expressão de genes relacionados à biossíntese do colesterol. A pesquisa também associou a exposição a um impacto importante na glicemia e na tolerância à glicose. A exposição ao BPA na gestação também pode estar relacionada com a predisposição a ingestão de doces nos descendentes. Estudos com animais de laboratório relacionaram a exposição a níveis ambientais de BPA a um aumento à preferência a doces nos machos e, consequentemente, a maior predisposição a obesidade futura ${ }^{79}$.

Uma associação de BPA com o ftalato di2-etil-exil ftalato (DEHP) utilizado na confecção de embalagens plásticas e o TBT utilizado como fungicida e algicida nas tintas que revestem embarcações, foi analisada para verificar a diferenciação de células-tronco mesenquimais de animais de laboratório expostos a essa mistura. Os resultados obtidos demonstraram que essa associação revelou ser mais obesogênica que o BPA isolado ${ }^{62}$. A mesma conclusão foi verificada na associação de BPA com o plastificante diciclo -orto-ftalato (DHCP) e o fungicida utilizado nas plantações de milho e soja, o tolifluanida (TF), provavelmente pelo efeito sinérgico entre as substâncias ${ }^{61,72}$.

\section{Ftalatos}

Os ftalatos são utilizados em plásticos para aumentar a flexibilidade e, portanto, são empregados na fabricação de muitos produtos, incluindo brinquedos e cosméticos. Representam essa classe o DEHP e seus metabolitos: o MEHP (Ftalato de Monoetilhexila) e o MEP (Mono Etil Ftalato). Além de apresentarem um risco potencial para a saúde reprodutiva, podem exercer efeitos sobre o metabolismo lipídico por ativação dos receptores PPAR $\gamma^{73}$.

Estudos epidemiológicos: Choi et al. ${ }^{48}$ analisando um conjunto de DE encontraram como resultado da pesquisa sobre obesidade na infância a presença 1,37 vezes maior de MEP na urina das crianças obesas em comparação com o grupo controle.

Estudos experimentais: Hao et al. ${ }^{66}$ realizaram pesquisas com a exposição perinatal de DEHP em animais de laboratório, e seus resultados sugerem que pode estar associada a um aumento da incidência de obesidade nos descendentes. Com relação à exposição ao MEHP, o efeito foi mais pronunciado nos machos que nas fêmeas. Adicionada a relação do DEHP com a obesidade, existe também a hipótese de que a fertilidade possa ser prejudicada pela exposição ambientalmente relevante desse composto ${ }^{73}$.

\section{Estrogênios}

Os estrogênios foram amplamente estudados com relação a distúrbios reprodutivos associados à exposição durante o período gestacional e, atualmente, têm demonstrado desempenhar um importante papel como obesogênicos. Nessa classe estão incluídos o estrógeno natural 17 estradiol $\left(\mathrm{E}_{2}\right)$, e os sintéticos etiniestradiol $\left(\mathrm{EE}_{2}\right)$ e DES como principais representantes. Através da excreção humana e de animais atingem os corpos hídricos conferindo poder estrogênico na água capaz de alterar o sistema reprodutivo e induzir a síntese da proteína vitelogenina nos peixes e causar efeitos à saúde humana, como redução na produção de espermatozoides, anomalias do sistema reprodutor masculino e feminino, aumento da incidência de câncer de mama, na vagina e na próstata, disfunção da tireoide, distúrbios metabólicos, entre outros efeitos ${ }^{9,14,20}$.

Estudos epidemiológicos: usando um biomarcador para quantificar a carga total de estrogênios, 490 placentas foram coletadas aleatoriamente para um estudo de coorte na Espanha, com o intuito de relacionar a estrogenicidade das 
amostras de placenta com o peso da criança ao nascer e o IMC ao completarem 14 meses. Os resultados sugerem que a exposição pré-natal pode aumentar o peso ao nascer dos meninos e que pode ter um impacto sobre a obesidade infantil $^{57}$. Segundo Hatch et al. ${ }^{51}$, o DES foi utilizado durante muitos anos como promotor de crescimento na produção de animais para consumo humano. Usando dados do Instituto Nacional de Câncer dos EUA, os pesquisadores avaliaram a associação entre DES e obesidade adulta a partir dos 20 anos até meados de vida, em 2.871 mulheres que foram expostas ao DES na fase pré-natal e 1.352 não expostas, dentro da faixa etária de 23 a 52 anos de idade. Os resultados obtidos sugerem que a exposição pré-natal ao DES pode estar associada a um pequeno aumento da obesidade adulta. A exposição ao E2 de origem exógena foi pesquisada em leitoas que receberam diferentes concentrações do hormônio durante a gestação. Apesar de a pesquisa ter objetivado avaliar baixas doses de exposição, os descendentes machos com 8 semanas apresentaram um aumento significativo na porcentagem de gordura total ${ }^{78}$.

\section{Alquilfenóis}

Os alquilfenóis são surfactantes não iônicos usados em uma grande variedade de produtos para uso doméstico e industrial, sendo que seus principais representantes são nonilfenol e octilfenol que agem sobre os receptores estrogênicos ${ }^{63}$

Estudos epidemiológicos: um estudo transversal foi realizado para investigar a associação entre alguns DE e a obesidade em meninas. Vários DE foram analisados, dentre eles nonilfenol, porém não houve associação entre os níveis desse composto e obesidade infantil ${ }^{48}$.

Estudos experimentais: a exposição perinatal de animais de laboratório ao nonilfenol foi utilizada para verificar os efeitos sobre o peso corporal, o IMC e o colesterol, e resultaram em associação positiva para aumento da predisposição à obesidade nos descendentes ${ }^{63}$.

\section{Agrotóxicos}

A ampla utilização de produtos químicos na agricultura em todo o mundo expõe crianças e adultos através de alimentos contaminados, água e solo a DE capazes de alterar seu desenvolvimento e reprodução. Agrotóxicos são DE potentes, se bioacumulam na cadeia alimentar, são persistentes no meio ambiente e seus efeitos deletérios no sistema reprodutivo de várias espécies animais já foi constatado ${ }^{14}$. O DDT e seus metabólitos são exemplos clássicos de agrotóxicos atuando como DE. Estudos de gestantes expostas ao DDT e seus metabólitos sugerem que além de provocarem distúrbios reprodutivos tenham também um poder obesogênico ${ }^{49}$. O herbicida 2,4-Diclorofenoxiacético (2,4-D), usado em todo o mundo, tem sido associado a várias alterações. Há estudos que mostram que os indivíduos que foram expostos ao 2,4-D têm baixa qualidade do esperma, enquanto que seus descendentes apresentam alterações no aparelho urinário. Estudos em humanos e animais sugerem uma relação entre a exposição a agrotóxicos e obesidade ${ }^{58,74,80-82}$.

Estudos epidemiológicos: utilizando os dados do estudo Chamacos para níveis séricos de DDT em gestantes, foi observado nos descendentes aos nove anos de idade um aumento de percentual de gordura nos meninos ${ }^{58}$. Na Dinamarca, um estudo mostrou que a exposição materna às combinações de pesticidas modernos, não persistentes, durante a gravidez de mulheres que trabalhavam em estufas, pode causar efeitos em longo prazo nos descendentes. Os resultados demonstraram que um maior nível de exposição materna no início da gravidez foi associado com menor peso da criança ao nascer, seguido de aumento do acúmulo de gordura corporal desde o nascimento até a idade escolar ${ }^{59}$. O herbicida 2,4-D, utilizado no mundo todo, apresentou poder obesogênico segundo uma pesquisa com adultos entre 20 e 85 anos nos EUA. O resultado deste estudo confirmou as conclusões dos pesquisadores em um trabalho anterior no qual relataram pela primeira vez a associação entre a presença na urina de um dos componentes do 2,4 D e obesidade entre crianças e adolescentes norte-americanas com idade variando entre seis e $19 \operatorname{anos}^{80}$.

Estudos experimentais: um estudo foi realizado para testar a hipótese de que o DDT promove a herança epigenética transgeracional da obesidade. Os resultados indicaram que a exposição durante a gestação pode promover obesidade e doenças associadas através de herança epigenética ${ }^{74}$.

\section{Alquilfenois - Hidrocarbonetos Aromáticos Policíclicos (HPA)}

São produtos químicos gerados a partir da combustão incompleta das matérias orgânicas. São cancerígenos e atuam como desreguladores endócrinos devido possivelmente à semelhança estrutural com estrogênios ${ }^{54,56}$.

Estudos epidemiológicos: estudo realizado com crianças afro-americanas e hispânicas nas- 
cidos nos EUA, cujas mães foram monitoradas na gravidez com relação à exposição aos HPA, obteve dados indicando que a exposição prénatal ao HPA está associada com a obesidade na infância ${ }^{54}$. Metabolitos urinários de HPA foram associados com aumento do IMC e da obesidade entre crianças de seis a 11 anos de idade, com associações menos expressivas nos adolescentes ${ }^{5}$.

\section{Discussão}

Na presente revisão, a obesidade foi abordada dentre importantes doenças associadas à exposição aos DE pelo fato de ser uma das principais preocupações de saúde publica no mundo todo, por estar acometendo cada vez mais crianças e adolescentes e devido à grande prevalência de doenças crônicas a ela associadas. A presença de contaminantes no ambiente pode interferir em múltiplos processos biológicos com relação à obesidade, podendo alterar o mecanismo de ação em relação ao gasto energético, distribuição e acúmulo do tecido adiposo e propiciar a herança epigenética ${ }^{83}$.

Os DE podem agir sobre a produção de leptina e adiponectina que são reguladores do equilíbrio energético do corpo, do metabolismo de glicose e lipídios e a resistência à insulina, atuando de forma distinta dependendo da fase da vida. A leptina regula o apetite e o peso corporal através da veiculação de sinais de saciedade para receptores hipotalâmicos. Em um adulto saudável é proporcional à gordura corporal, fazendo com que dessa maneira a leptina se torne um marcador de obesidade tanto na infância quanto na fase adulta.

A adiponectina é também um regulador metabólico que apresenta níveis mais baixos em indivíduos obesos ou com resistência à insulina ${ }^{84,85}$. A atividade da aromatase, enzima que converte testosterona em $\mathrm{E}_{2}$, pode ser afetada pelos DE por interferir na ação de hormônios tireoidianos ou contribuir com a obesidade através da atuação como agonista sobre os receptores PPAR $\gamma^{36}$. A ativação inadequada dos receptores $\operatorname{PPAR} \gamma$ leva ao aumento da diferenciação de adipócitos, alterando o armazenamento de energia, e a resistência a insulina. Os ftalatos são exemplos de agonistas de PPAR $\gamma$ e estão associados ao aumento da circunferência da cintura ${ }^{35,86}$. Os fatores que controlam o apetite e o gasto energético estão integrados no eixo hipotálamo-pituitária-adrenal. O hipotálamo é o centro de equilíbrio de energia e apetite, que pode ser afetado pela ação dos $\mathrm{DE}^{87}$.
As mudanças epigenéticas durante o desenvolvimento acarretam impactos duradouros sobre sistemas metabólicos podendo provocar efeitos transgeracionais ${ }^{88}$. Skinner et al. ${ }^{74}$ relataram a exposição ao DDT no período gestacional em animais de laboratório, sendo que somente a geração F3 desenvolveu obesidade em mais de 50\% dos descendentes. BPA e DES também podem induzir a herança gestacional ${ }^{40,60,62}$.

Nem todos os mecanismos de ação estão esclarecidos e avaliar a exposição para possível associação com a obesidade é bastante complexo. A exposição ambiental geralmente ocorre com uma mistura de vários DE, exigindo um conhecimento amplo de farmacocinética, interações entre substâncias, biotransformação, para auxiliar nas avaliações ${ }^{89}$. Podem interferir nos resultados das pesquisas fatores que predispõe o surgimento da obesidade como dieta e atividade física. A excreção e o metabolismo dos DE podem ser influenciados por uma maior atividade física, ou por abuso de alimentos com alto teor de gordu$\mathrm{ra}^{90}$. Apesar de as causas comuns do aumento da obesidade no mundo serem as dietas hipercalóricas e a vida sedentária, um estudo realizado com peixes zebra utilizando água de lagos na Noruega com concentrações ambientalmente relevantes de DE demonstrou que a poluição ambiental afetou a homeostase do peso e a sinalização da insulina de peixes que estiveram em contato com a água coletada ${ }^{68}$.

Análises equivocadas podem ocorrer por outros fatores, como a relação entre DE, sexo dos envolvidos e acumulo de tecido adiposo. De maneira geral, os DE têm a tendência de se acumularem nos tecidos adiposos devido ao seu caráter lipossolúvel ${ }^{5}$, fazendo com que as pessoas obesas possam acumular mais DE do que as não obesas. Pacientes obesos submetidos a cirurgia bariátrica em Portugal foram analisados com relação à presença de DE no tecido adiposo visceral e subcutâneo, e os dados demonstraram a presença da substância em ambos os tecidos em 96,3\% dos indivíduos pesquisados ${ }^{52}$, entretanto, não é possível confirmar se os DE causaram a obesidade ou se houve uma maior concentração devido ao acumulo de tecido adiposo.

Segundo Fernandez et al..$^{91}$, existe a possibilidade de que o BPA detectado nos indivíduos pesquisados ocorreu devido ao aumento da gordura corporal, ao invés de ter induzido o aumento do IMC. Os testes com tecido adiposo humano realizados in vitro não encontraram associação entre concentração de BPA e IMC, indicando que o aumento de BPA pode não ter relação com o de 
células adiposas. Volberg et al. ${ }^{42}$, analisando várias pesquisas sobre BPA e obesidade, alegam que os dados são limitados devido à natureza transversal dos estudos, não sendo possível avaliar se a exposição precedeu o desenvolvimento da obesidade ou se foi um reflexo dela.

O acumulo de gordura também sofre distribuição diferenciada entre os sexos e sinaliza que a fisiologia do tecido adiposo sofre influência de hormônios sexuais endógenos, incluindo $\mathrm{E}_{2} \mathrm{e}$ testosterona, e por essa razão pode se comportar de maneira diferente diante dos $\mathrm{DE}^{92}$. Esse comportamento foi observado por Warner et al. ${ }^{58}$, que verificaram concentrações mais elevadas de DDT no pré-natal associadas significativamente com aumento de IMC e circunferência da cintura apenas nos meninos.

As pesquisas durante o período gestacional podem ser uma fonte de viés, já que nesse período podem ocorrer alterações fisiológicas normais ou fatores que podem alterar o desenvolvimento do feto sem que haja relação com a exposição ${ }^{93}$. O organismo em desenvolvimento tem uma maior taxa metabólica quando comparado com um adulto que, em alguns casos, pode resultar num aumento dos efeitos adversos. Fetos e recém-nascidos são extremante sensíveis aos DE, principalmente os que apresentam caráter estrogênico ${ }^{94}$. O metabolismo de cada substância pode interferir nos resultados, como o caso do BPA, cujo metabolismo e excreção são rápidos em decorrência de uma meia vida de aproximadamente seis horas em seres humanos e, portanto, as amostras podem não refletir a realidade da exposição ${ }^{95}$.

A análise dos resultados pode ser dificultada ainda devido à relação não linear entre dose de exposição e desfecho; várias matrizes para detectar um mesmo DE como a urina, o sangue total, o sangue do cordão umbilical, além da ausência de estudos estratificados com relação a machos e fêmeas, e devido a resultados contraditórios com relação ao $\operatorname{sexo}^{90}$. Apesar do exposto, segundo Newbold et al. ${ }^{94}$, não se pode assumir que o sobrepeso e a obesidade sejam escolhas pessoais simplesmente, como atividade física e alimentação, mas considerar que os eventos complexos, incluindo substâncias químicas ambientais possam estar envolvidas.

A extensão das consequências para a saúde de um sistema endócrino alterado por qualquer que seja o DE ainda é desconhecida e preocupante, principalmente pelo fato dos organismos em desenvolvimento serem os mais afetados devido à extrema sensibilidade a esses compostos ${ }^{94}$. A precaução é necessária até que novas pesquisas possam elucidar os reais efeitos adversos causados pela exposição aos DE. Podemos evitar a utilização de produtos confeccionados com plásticos que contenham BPA, utilizar alimentos orgânicos livres de agrotóxicos, mas somos dependentes da água cada vez mais contaminada por produtos químicos.

Frear o avanço da cadeia produtiva e conter o consumo são tarefas desafiadoras, sendo importante a busca de outros mecanismos para evitar o contato com esses produtos. A literatura mostra que os esgotos domésticos e industriais representam uma importante fonte de contaminação dos corpos hídricos e que as ETE não removem satisfatoriamente os $\mathrm{DE}^{22,23}$. Pesquisas realizadas no Brasil mostram que $\mathrm{DE}$, tais como nonilfenol, BPA, HPA e estrogênios já foram identificados em efluentes de ETE e águas superficiais ${ }^{96}$. Importantes ações podem ser realizadas para prevenir que a água contendo DE retorne às torneiras. Processos avançados de remoção de contaminantes nas ETE têm demonstrado eficiência na remoção de DE, porém a adoção desses sistemas complementares de tratamento envolve dificuldades como os investimentos necessários para a implantação, sendo este um fator limitan$\mathrm{te}^{25}$. Existe uma preocupação global na busca de processos de remoção completa dos DE, já que podem agir mesmo em doses diminutas, e que apresentem viabilidade técnica e econômica.

\section{Conclusão}

A revisão sugere que a exposição a determinados DE, principalmente na fase intrauterina, está associada com a obesidade. As pesquisas relacionadas ao tema necessitam de resultados mais consistentes apesar do grande avanço nessa área na última década. Ainda existem muitas lacunas a serem esclarecidas e os efeitos relacionados à exposição no longo prazo ainda são escassos, porém não existe dúvida da importância de se tomar medidas preventivas que diminuam o acesso, o consumo e o descarte e que se faça o tratamento sanitário desses compostos. Ao se utilizar processos eficientes para a remoção de produtos químicos dos efluentes domésticos e industriais, estamos contribuindo significativamente para minimizar os efeitos adversos dos DE que causam danos tanto para a fauna e flora presente nos corpos hídricos quanto para o homem. 
Colaboradores

RCN Pontelli, AA Nunes e SVWB Oliveira participaram igualmente de todas as etapas de elaboração do artigo.

\section{Referências}

1. Brandt EMF. Avaliação da remoção de fármacos e desreguladores endócrinos em sistemas simplificados de tratamento de esgoto (reatores UASB seguidos de pós-tratamento) [dissertação]. Belo Horizonte: Universidade Federal de Minas Gerais; 2012.

2. Agência Nacional de Águas (ANA). [Internet] 2012. [acessado 2015 maio 15]. Disponível em: http://arquivos.ana.gov.br/imprensa/arquivos/Conjuntura 2012. pdf

3. Brasil. Ministério das Cidades. Sistema Nacional de Informações sobre Saneamento - SNIS [ Internet]. 2013. [acessado 2015 maio 23]. Disponível em: http://www. snis.gov.br/PaginaCarrega.php?EWRErterterTERTer $=105$.

4. Aquino SF, Brandt EMF, Chernicharo CAL. Remoção de fármacos e desreguladores endócrinos em estações de tratamento de esgoto: revisão da literatura. Eng Sanit Ambient 2013; 18(3):187-204.

5. Ghiselli G, Jardim WF. Interferentes endócrinos no ambiente. Química Nova 2007; 30:695-706.

6. Clement CR, Colborn T. Herbicides and fungicides: a perspective on potential human exposure. In: CobornT, Clement CR, editors. Chemically-induced alterations in sexual and functional development: The wildlife/ human connection. Princeton: Princeton Scientific Pub; 1992. p. 347-364.

7. Soeiro PIdSP. Disruptores endócrinos. [Internet] 2014. [acessado 2015 abr 17]. Disponível em: https://estudogeral.sib.uc.pt/jspui/bitstream/10316/28605/1/Tese.pdf

8. Baird C. Química ambiental. Porto Alegre: Bookman; 2002.

9. Lintelman J, Katayama A, Kurihara N, Shore L, Wenzel A. Endocrine disruptors in the environment. Pure Appl Chem 2003; 75:631.

10. Frye CA, Bo E, Calamandrei G, Calzà L, Dessì-Fulgheri F, Fernández M, Fusani L,Kah O, Kajta M, Le Page Y, Patisaul HB, Venerosi A, Wojtowicz AK, Panzica GC.Endocrine disrupters: a review of some sources, effects, and mechanisms of actions on behaviour and neuroendocrine systems. J Neuroendocrinol 2012; 24(1):144159.

11. Jolly C, Katsiadaki L, Le Belle N, Mayer L, Dufour S. Development of a stickleback kidney cell culture assay for the screening of androgenic and anti-androgenic endocrine disrupters. Aquat Toxicol 2006; 79:158-166.

12. Environmental Protection Agency (EPA). Occurrence of contaminants of emerging concern in wastewater from nine publicly owned treatment works. [Internet].2009. [acessado 2015 maio 20]. Disponível em: http://water.epa.gov/scitech/swguidance/ppcp/upload/2009_08_07_ppcp_studies_9potwstudy.pdf

13. Colborn T, Vom Saal FS, Soto AM. Developmental effects of endocrine disrupting chemicals in wildlife and humans. Environ Health Perspect 1993; 101:378-384

14. Birkett JW, Lester JN. Endocrine disrupters in wastewater and sludge treatment process. Boca Raton: Lewis/ CRC Press; 2003.

15. Diamanti-Kandarakis E, Bourguignon JP, Giudice LC, Hauser R, Prins GS, Soto AM,Zoeller RT, Gore AC. Endocrine-disrupting chemicals: an Endocrine society scientific statement. Endocr Rev 2009; 30(4):293-342. 
16. Vandenberg LN, Colborn T, Hayes TB, Heindel JJ, Jacobs DR Jr, Lee DH, Shioda T, Soto AM, vom Saal FS, Welshons WV, Zoeller RT, Myers JP. Hormones and endocrine disrupting chemicals: low-dose effects and non monotonic dose responses. Endocr Rev 2012; 33(3):378-455.

17. DiVall SA. The influence of endocrine disruptors on growth and development of children. Curr Opin Endocrinol Diabetes Obesity 2013; 20(1):50-55.

18. Casals-Casas C, Desvergne B. Endocrine disruptors: from endocrine to metabolic disruption. Annu Rev Physiol 2011; 73:135-162.

19. Weiss B. The intersection of neurotoxicology and endocrine disruption. Neurotoxicology 2012; 33(6):14101419.

20. Bila D, Montalvao AF, Azevedo DdA, Dezotti M. Estrogenic activity removal of 17 beta-estradiol by ozonation and identification of by-products. Chemosphere 2007; 69(5):736-746

21. Pereira RdO, Carminato VM, Vieira EM, Daniel LA. Partial degradation of 17 beta-estradiol by the chlorination applied to the treatment of water. Eng Sanit Ambient 2013; 18(3):215-222.

22. Chen CY, Wen TY, Wang GS, Cheng HW, LinYH, Lien GW. Determining estrogenic steroids in Taipei waters and removal in drinking water treatment using highflow solid-phase extraction and liquid chromatography/tandem mass spectrometry. Sci Total Environ 2007; 378(3):352-365.

23. Liu ZH, Kanjo Y, Mizutani S. Removal mechanisms for endocrines disrupting compounds (EDCs) in wastewater treatment - physical means, biodegration and chemical advanced oxidation: a review. Sci Total Environ 2009; 407:731-748.

24. Baronti C, Curini R, D’Ascenzo G, Corcia A, Di Gentili A, Samperi R.Monitoring natural and synthetic estrogens at activated sludge sewage treatment plants and in a receiving river water. Environ Sci Tecnol 2000; 34(24):5059-5066.

25. Raimundo C. Ocorrência de interferentes endócrinos e produtos farmacêuticos nas águas superficiais da bacia do rio Atibaia [dissertação]. Campinas: Universidade Estadual de Campinas; 2007.

26. Esteves V, Otero M, Silva CP. Processes for the elimination of estrogenic steroid hormones from water: Environ Pollut 2012; 38:165

27. Marinho BA. Estudo da potencialidade da fotocatálise heterogênea e dos processos fenton para degradação de micropoluentes em águas residuárias (esgoto tratado) [dissertação]. Curitiba: Universidade federal do Paraná; 2013.

28. Newbold RR, Padilla-Banks E, Jefferson WN, Heindel JJ. Effects of endocrine disruptors on obesity. Int J Androl 2008; 31(2):201-208

29. Romano ME, Savitz DA, Braun JM. Challenges and future directions to evaluating the association between prenatal exposure to endocrine disrupting chemicals and childhood obesity. Curr Epidemiol Rep 2014; 1(2):57-66

30. Janesick A, Blumberg B. Endocrine disrupting chemicals and the developmental programming of adipogenesis and obesity. Birth Defects Res C Embryo Today 2011; 93:34-50.
31. Legler J, Fletcher T, Govarts E, Porta M, Blumberg B, Heindel JJ, Trasande L. Obesity, diabetes, and associated costs of exposure to endocrine-disrupting chemicals in the European Union. Endocrinol Metab 2015; 100(4):1278-1288.

32. Baillie-Hamilton PF. Chemical toxins: a hypothesis to explain the global obesity epidemic. J Altern Complement Med 2002; 8(2):185-192.

33. Casals-Casas C, Desvergne B. Endocrine disruptors: From endocrine to metabolic disruption. Ann Rev Physiol 2011; 73:135-162.

34. Chamorro-García R, Blumberg B. Transgenerational effects of obesogens and the obesity epidemic. Curr Opin Pharmacol. 2014; 19:153-158.

35. Grün F, Blumberg B. Perturbed nuclear receptor signaling by environmental obesogens as emerging factors in the obesity crisis. Rev Endocr Metab Disord 2007; $8(2): 161-171$

36. Janesick A, Blumberg B. Minireview: PPAR gamma as the target of obesogens. Journal of Steroid Biochemistry and Molecular Biology. 2011; 127(1-2):4-8.

37. Gregoire FM. Adipocyte differentiation: from fibroblast to endocrine cell. Exp Biol Med (Maywood) 2001; 226:997-1002.

38. Saal F, Akingbemi B, Belcher S, BIrnbaum L, Crain D, Eriksen M. Chapel Hill.Bisphenol A Expert Panel consensus statement: integration of mechanisms, effects in animals and potential to impact human health at current levels of exposure. Reprod Toxicol 2007; 24:1-26.

39. Rosenfeld CS, Sieli PT, Warzak DA, Ellersieck MR, Pennington KA, Roberts RM. Maternal exposure to bisphenol A and genistein has minimal effect on $\mathrm{A}(\mathrm{vy}) / \mathrm{a}$ offspring coat color but favors birth of agouti over nonagouti mice. Proc Natl Acad Sci U S A. 2013; 110 (2):537-542

40. Dolinoy DC, Huang D, Jirtle RL. Maternal nutrient supplementation counteracts bisphenol A-induced DNA hypomethylation in early development. Proc Natl Acad Sci U S A. 2007; 104(32):13056-13061.

41. Tang W-y, Ho S-m. Epigenetic reprogramming and imprinting in origins of disease. Rev Endocr Metab Disord 2007; 8(2):173-182.

42. Volberg V, Harley K, Calafat AM, Davé V, McFadden J, Eskenazi B, Holland N. Maternal bisphenol a exposure during pregnancy and its association with adipokines in Mexican-American children. Environ Mol Mutagen 2013; 54(8):621-628

43. Li DK, Miao M, Zhou Z, Wu C, Shi H, Liu X, Wang S, Yuan W. Urine bisphenol-A level in relation to obesity and overweight in school-age children. PLoS One 2013; 8(6):e65399.

44. Center for the Heath Assessment of Mothers and Children of Salinas (C.H.A.M.A.C.O.S.). CHAMACOS Study. [Internet]. [acessado 2015 jun 27]. Disponível em: http://cerch.org/research-programs/chamacos

45. Harley KG, Schall RA, Chevrier J, Tyler K, Aguirre H, Bradman A, Holland NT, Lustig RH, Calafat AM, Eskenazi B. Prenatal and postnatal bisphenol A exposure and body mass index in childhood in the CHAMACOS cohort. Environ Health Perspect 2013; 121(4):514-520.

46. Miao MH, Yuan W, Zhu GP, He XF, Li DK. In utero exposure to bisphenol-A and its effect on birth weight of offspring. Reprod Toxicol 2011; 32(1):64-68. 
47. Carwile JL, Michels KB. Urinary bisphenol A and obesity: NHANES 2003-2006. Environ Res 2011; 111(6):825-830.

48. Choi J, Eom J, Kim J, Lee S, Kim Y. Association between some endocrine-disrupting chemicals and childhood obesity in biological samples of young girls: A cross-sectional study. Environ Toxicol Pharmacol 2014 38(1):51-57.

49. Elobeid MA, Padilla MA, Brock DW, Ruden DM, Allison DB. Endocrine disruptors and obesity: An examination of selected persistent organic pollutants in the NHANES (1999-2002). Int J Environ Res Public Heal 2010; 7(7):2988-3005

50. Gauthier MS, Rabasa-Lhoret R, Prud'homme D, Karelis AD, Geng D, Van Bavel B, Ruzzin J. The metabolically healthy but obese phenotype is associated with lower plasma levels of persistent organic pollutants as compared to the metabolically abnormal obese phenotype. J Clin Endocrinol Metab 2014; 99(6):E1061-E1066.

51. Hatch EE, Troisi R, Palmer JR, Wise LA, Titus L, Strohsnitter WC, Ricker W, Hyer M, Hoover RN. Prenatal diethylstilbestrol exposure and risk of obesity in adult women. J Dev Orig Health Dis 2015; 6(3):201207.

52. Pestana D, Faria G, Sá C, Fernandes VC, Teixeira D, Norberto S, Faria A, Meireles M, Marques C, CorreiaSá L, Cunha A, Guimarães JT, Taveira-Gomes A, Santos AC, Domingues VF, Delerue-Matos C, Monteiro R, Calhau C. Persistent organic pollutant levels in human visceral and subcutaneous adipose tissue in obese individuals-Depot differences and dysmetabolism implications. Environ Res 2014; 133:170-177.

53. Rönn M, Lind L, Örberg J, Kullberg J, Söderberg S, Larsson A, Johansson L, Ahlström H, Lind PM. Bisphenol A is related to circulating levels of adiponectin, leptin and ghrelin, but not too fat mass or fat distribution in humans. Chemosphere 2014; 112:42-48.

54. Rundle A, Hoepner L, Hassoun A, Oberfield S, Freyer G, Holmes D, Reyes M, Quinn J, Camann D, Perera F, Whyatt R. Association of childhood obesity with maternal exposure to ambient air polycyclic aromatic hydrocarbons during pregnancy. Am J Epidemiol 2012; 175(11):1163-1172.

55. Savastano S, Tarantino G, D'Esposito V, Passaretti F, Cabaro S, Liotti A, Liguoro D, Perruolo G, Ariemma F, Finelli C, Beguinot F, Formisano P, Valentino R Bisphenol-A plasma levels are related to inflammatory markers, visceral obesity and insulin-resistance: a cross-sectional study on adult male population. J Transl Med 2015; 13:169.

56. Scinicariello F, Buser MC. Urinary polycyclic aromatic hydrocarbons and childhood obesity: NHANES (20012006). Environ Health Perspect 2014; 122(3):299-303.

57. Vilahur N, Molina-Molina JM, Bustamante M, Murcia M, Arrebola JP, Ballester F, Mendez MA, Garcia-Esteban R, Guxens M, Santa Marina L, Tardón A, Sunyer J, Olea N, Fernandez MF. Male specific association between xenoestrogen levels in placenta and birthweight. Environ Intl 2013; 51:174-181.
58. Warner M, Wesselink A, Harley KG, Bradman A, Kogut K, Eskenazi B. Prenatal exposure to dichlorodiphenyltrichloroethane and obesity at 9 years of age in the CHAMACOS study cohort. Am J Epidemiol 2014; 179(11):1312-1322.

59. Wohlfahrt-Veje C, Main KM, Schmidt IM, Boas M, Jensen TK, Grandjean P, Skakkebæk NE, Andersen HR. Lower birth weight and increased body fat at school age in children prenatally exposed to modern pesticides: a prospective study. Environ Health 2011; 10(1):79.

60. Xue J, Wu Q, Sakthivel S, Pavithran PV, Vasukutty JR, Kannan K. Urinary levels of endocrine-disrupting chemicals, including bisphenols, bisphenol A diglycidyl ethers, benzophenones, parabens, and triclosan in obese and non-obese Indian children. Environ Res 2015; 137:120-128.

61. Bastos Sales L, Kamstra JH, Cenijn PH, van Rijt LS, Hamers T, Legler J. Effects of endocrine disrupting chemicals on in vitro global DNA methylation and adipocyte differentiation. Toxicol in Vitro 2013; 27(6):1634-1643.

62. Biemann R, Fischer B, Navarrete Santos A. Adipogenic effects of a combination of the endocrine-disrupting compounds bisphenol a, diethylhexylphthalate, and tributyltin. Obes Facts 2014; 7(1):48-56

63. Hao CJ, Cheng XJ, Xia HF, Ma X. The endocrine disruptor 4-nonylphenol promotes adipocyte differentiation and induces obesity in mice. Cell Physiol Biochem 2012; 30(2):382-394

64. Hao CJ, Cheng XJ, Xia HF, Ma X. The endocrine disruptor diethylstilbestrol induces adipocyte differentiation and promotes obesity in mice. Toxicol Appl Pharmacol 2012; 263(1):102-110.

65. Hao CJ, Cheng XJ, Xia HF, Ma X. The endocrine disruptor mono-(2-ethylhexyl) phthalate promotes adipocyte differentiation and induces obesity in mice. Biosci Rep 2012; 32(6):619-629

66. Hao C, Cheng X, Guo J, Xia H, Ma X. Perinatal exposure to diethyl-hexyl-phthalate induces obesity in mice. Front Biosci 2013; 5:725-733.

67. Kamstra JH, Hruba E, Blumberg B, Janesick A, Mandrup S, Hamers T, Legler J. Transcriptional and epigenetic mechanisms underlying enhanced in vitro adipocyte differentiation by the brominated flame retardant bde-47. Environ Sci Technol 2014; 48(7):4110-4119.

68. Lyche JL, Nourizadeh-Lillabadi R, Karlsson C, Stavik B, Berg V, Skåre JU, Alestrøm P, Ropstad E. Natural mixtures of POPs affected body weight gain and induced transcription of genes involved in weight regulation and insulin signaling. Aquat Toxicol 2011; 102(34):197-204

69. Marmugi A, Lasserre F, Beuzelin D, Ducheix S, Huc L, Polizzi A, Chetivaux M, Pineau T, Martin P, Guillou H, Mselli-Lakhal L. Adverse effects of long-term exposure to bisphenol A during adulthood leading to hyperglycaemia and hypercholesterolemia in mice. Toxicology 2014; 325:133-143.

70. Neel BA, Brady MJ, Sargis RM. The endocrine disrupting chemical tolylfluanid alters adipocyte metabolism via glucocorticoid receptor activation. Mol Endocrinol 2013; 27(3):394-406. 
71. Pereira-Fernandes A, Demaegdt H, Vandermeiren K, Hectors TLM, Jorens PG, Blust R, Vanparys C. Evaluation of a Screening System for Obesogenic Compounds: Screening of Endocrine Disrupting Compounds and Evaluation of the PPAR Dependency of the Effect. PLoS ONE 2013; 8(10):e77481:1-17.

72. Sargis RM, Johnson DN, Choudhury RA, Brady MJ. Environmental endocrine disruptors promote adipogenesis in the 3T3-L1 cell line through glucocorticoid receptor activation. Obesity 2010; 18(7):1283-1288.

73. Schmidt JS, Schaedlich K, Fiandanese N, Pocar P, Fischer B. Effects of Di(2-ethylhexyl) phthalate (DEHP) on female fertility and adipogenesis in $\mathrm{C} 3 \mathrm{H} / \mathrm{N}$ Mice. Environ Health Perspect 2012; 120(8):1123-1129.

74. Skinner MK, Manikkam M, Tracey R, Guerrero-Bosagna $C$, Haque M, Nilsson EE. Ancestral dichlorodiphenyltrichloroethane (DDT) exposure promotes epigenetic transgenerational inheritance of obesity. BMC Med 2013; 11:228

75. Tracey R, Manikkam M, Guerrero-Bosagna C, Skinner MK. Hydrocarbons (jet fuel JP-8) induce epigenetic transgenerational inheritance of obesity, reproductive disease and sperm epimutations. Reprod Toxicol 2013; 36:104-116

76. Van Esterik JC, Dolle ME, Hodemaekers-Goossens HM, Imholz S, van Leeuwen SP, Legler J, van der Ven LTM. Perinatal programming of obesity later in life by the environmental endocrine disruptor bisphenol $\mathrm{A}$ in a mouse model. Toxicol Lett 2011; 205:S38-S.

77. Wei J, Lin Y, Li YY, Ying CJ, Chen J, Song LQ, Zhou Z, Lv Z, Xia W, Chen X, Xu S. Perinatal Exposure to Bisphenol A at Reference Dose Predisposes Offspring to Metabolic Syndrome in Adult Rats on a High-Fat Diet. Endocrinology 2011; 152(8):3049-3061.

78. Werner Fürst R, Pistek VL, Kliem H, Skurk T, Hauner H, Meyer HHD, Ulbrich SE. Maternal low-dose estradiol-17 $\beta$ exposure during pregnancy impairs postnatal progeny weight development and body composition. Toxicol Appl Pharmacol 2012; 263(3):338-344.

79. Xu X, Tan L, Himi T, Sadamatsu M, Tsutsumi S, Akaike $\mathrm{M}$, Kato N. Changed preference for sweet taste in adulthood induced by perinatal exposure to bisphenol A: a probable link to overweight and obesity. Neurotoxicol Teratol 2011; 33(4):458-463.

80. Wei Y, Zhu J, Nguyen A. Urinary concentrations of dichlorophenol pesticides and obesity among adult participants in the US National Health and Nutrition Examination Survey (NHANES) 2005-2008. International Journal of Hygiene and Environmental Health 2014; 217(2-3):294-299.

81. Alves MG, Neuhaus-Oliveira A, Moreira PI, Socorro S, Oliveira PF. Exposure to 2,4-dichlorophenoxyacetic acid alters glucose metabolism in immature rat Sertoli cells. Reproductive Toxicology 2013; 38:81-88.

82. Valvi D, Mendez MA, Martinez D, Grimalt JO, Torrent M, Sunyer J, Vrijheid M. Prenatal Concentrations of Polychlorinated Biphenyls, DDE, and DDT and Overweight in Children: A Prospective Birth Cohort Study. Environ Health Perspect 2012; 120(3):451-7.
83. Gruen F, Blumberg B. Minireview: The Case for Obesogens. Mol Endocrinol 2009; 23(8):1127-1134.

84. McMillen IC, Edwards LJ, Duffield J, Muhlhausler BS. Regulation of leptin synthesis and secretion before birth: implications for the early programming of adult obesity. Reproduction 2006; 131(3):415-427.

85. Gil-Campos M, Canete R, Gil A. Adiponectin, the missing link in insulin resistance and obesity. Clin Nutr 2004; 23(5):963-974.

86. Hurst $\mathrm{CH}$, Waxman DJ. Activation of PPAR alpha and PPAR gamma by environmental phthalate monoesters. Toxicol Sci 2003; 74(2):297-308.

87. de Cock M, van de Bor M. Obesogenic effects of endocrine disruptors, what do we know from animal and human studies? Environ Int 2014; 70:15-24.

88. Janesick A, Blumberg B. Obesogens, stem cells and the developmental programming of obesity. Int J Androl 2012; 35(3):437-448

89. Johns DO, Stanek LW, Walker K, Benromdhane S, Hubbell B, Ross M, Devlin RB, Costa DL, Greenbaum DS. Practical Advancement of Multipollutant Scientific and Risk Assessment Approaches for Ambient Air Pollution. Environ Health Perspect 2012; 120(9):1238-1242.

90. Romano ME, Savitz DA, Braun JM. Challenges and future directions to evaluating the association between prenatal exposure to endocrine disrupting chemicals and childhood obesity. Curr Epidemiol Rep 2014; 1(2):57-66.

91. Fernandez MF, Arrebola JP, Taoufiki J, Navalon A, Ballesteros O, Pulgar R, Vilchez JL, Olea N. Bisphenol-A and chlorinated derivatives in adipose tissue of women. Reprod Toxicol 2007; 24(2):259-264.

92. Wells JCK. Sexual dimorphism of body composition. Best Practice \& Research Clinical Endocrinology \& Metabolism 2007; 21(3):415-430.

93. Li D, Longnecker MP, Dunson DB. Lipid Adjustment for Chemical Exposures Accounting for Concomitant Variables. Epidemiology 2013; 24(6):921-928.

94. Newbold RR, Padilla-Banks E, Snyder RJ, Phillips TM, Jefferson WN. Developmental exposure to endocrine disruptors and the obesity epidemic. Reproductive Toxicology 2007; 23(3):290-296.

95. Dekant W, Voelkel W. Human exposure to bisphenol A by biomonitoring: Methods, results and assessment of environmental exposures. Toxicology and Applied Pharmacology 2008; 228(1):114-134.

96. Bila DM, Dezotti M. Desreguladores endócrinos no meio ambiente: efeitos e conseqüências. Quím Nova 2007; 30(3):651-666.

Artigo apresentado em 20/08/2015

Aprovado em 04/12/2015

Versão final apresentada em 17/12/2015 